\title{
Experimental-laboratory study of the flow around mussel shocks
} George Pechlivanidis ${ }^{1}$, Alexandros Antoniou ${ }^{2}$, Yiannis Savvidis ${ }^{3 *}$

Department of Civil Engineering T.E., Alexander Technological Institute of Thessaloniki, Greece *Corresponding Author Email: savvidis@aqua.teithe.gr

\begin{abstract}
The productivity in an organized mussel culture area is closely related to the hydrodynamics in the area where the mussel units are located. The interaction between the hydrodynamics and mussel farming in Chalastra (NW Thessalonikigulf) has been investigated during last decades. In the framework of the study of optimizing the quality of mussels production in mussel farming areas, a laboratory channel was designed, where the flow around and possibly through the mussel shocks would be studied in physical scale. The experiments were designed in physical/natural scale and the relevant variables were determined. Moreover, the specific positions for the measurements, the depth of the flow and the velocity currents were also determined. The following three mean velocity values of entrance water velocity $U$ were used in the experiment; $5 \mathrm{~cm} / \mathrm{sec}, 7 \mathrm{~cm} / \mathrm{sec}$ and $9 \mathrm{~cm} / \mathrm{sec}$. A basic research parameter used in the experiment was the distance between the mussel shocks. Four cases were taken into account: 300mm, 500mm, 700mm and 900mm. The final goal was the determination of the velocity field in the areas around the shocks. The velocity field was studied with the modern Particle Image Velocimetry technique. According to the above presented experiments, for distances between the shocks greater than $500 \mathrm{~mm}$ the velocity field is almost restored. Furthermore the case of larger distance between the shocks (i.e. $90 \mathrm{~cm})$ present the largest percentage of the velocity class $5-10 \mathrm{~cm} / \mathrm{sec}$ (occurring for entrance current velocities 7 and $9 \mathrm{~cm} / \mathrm{s}$ ) which seem to be the best range for mussel's growth.
\end{abstract}

Keywords - current velocity, laboratory experiments, mussel shocks, PIV method.

\section{INTRODUCTION}

It is well known that the productivity in a mussel farm is closely related to the hydrodynamics in the area. That significant factor, i.e. the hydrodynamics in a mussel farm has been extensively investigated during last decades in the coastal area of Chalastra (W-NW gulf of Thessaloniki, North Greece). Thus, such relevant studies for the specific area started to take place, in a more insistent and organized way, at the beginning of the $21^{\text {st }}$ century. More specifically, [1] realized field measurements in the area which showed that currents are quite weak in the area of the mussel farms. Galinou-Mitsoudi et al. [2] later worked on a mathematical simulation showing that field measurements, mentioned before, were very well approximated by the results of a coarse hydrodynamic model. [3], [4] and [5] conducted field and numerical experiments while [6]and [7]studied mussel cultures and hydrodynamics with the help of mathematical simulations and the development of general management tools. These afore mentioned research works finally led to the findings listed below: (a) the largest proportions of current speeds ( $>40 \%$ ) were recorded between 0 and $5 \mathrm{~cm} / \mathrm{s}$, which corresponded to very weak currents, suitable only for low density farming, (b) the longest treatment configuration (from the four configurations corresponding to distance between shocks 30 or 50 or 70 or $90 \mathrm{~cm}$ )i.e. shock distance $90 \mathrm{~cm}$, led to the larger values of current speeds $(90 \mathrm{~cm}$ treatment had the largest percentage for the class $5-10 \mathrm{~cm} / \mathrm{sec}$ which is the most suitable current speed for the mussel farming activity according to [8] (c) there was a great variability of the current direction at the long line level and (d) it was observed that sometimes the current, moving towards the mussel unit deviates its route and is not entering inside the farm, which may lead to less food availability inside the farm.[9] in the framework of his doctoral research conducted both field and laboratory experiments however the latter experiments were based on the use of cylinders. The flow over two in-line cylinders in laminar and turbulent flows was also studied with the help of numerical simulations by [10].Furthermore, [11] studied the turbulent-flow characteristics and the mechanism of vortex shedding behind one and two square obstacles centered inside a 2-D channel. The study was based on the use of large eddy simulation and finite-element technique.

Taking into account the aforementioned research works, the need of a more detailed investigation on the hydrodynamics related to the mussel shocks, in a small scale, laboratory level was arisen. More specifically, it was considered that this research could take place in a laboratory channel with natural shocks from the sea field so that the experiments would lead to the best possible integrity of the results. In the framework of the study of optimizing the quality of mussels production in mussel farming areas, a laboratory structure (channel) was designed, where the flow around and possibly through the mussel 
shocks would be studied in physical (natural) scale. Therefore, an extension of the already existed channel in the hydraulic laboratory of Civil Eng. Dept. of ATEITh (Alexander Technological Educational Institute of Thessaloniki) was realized. More specifically, the old channel length increased from 4 to $10 \mathrm{~m}$, while the dimensions of a cross section (width and height) of the channel were $50 \mathrm{~cm}$ x $50 \mathrm{~cm}$, with the ability of variable inclination of the channel's floor (Fig 1).

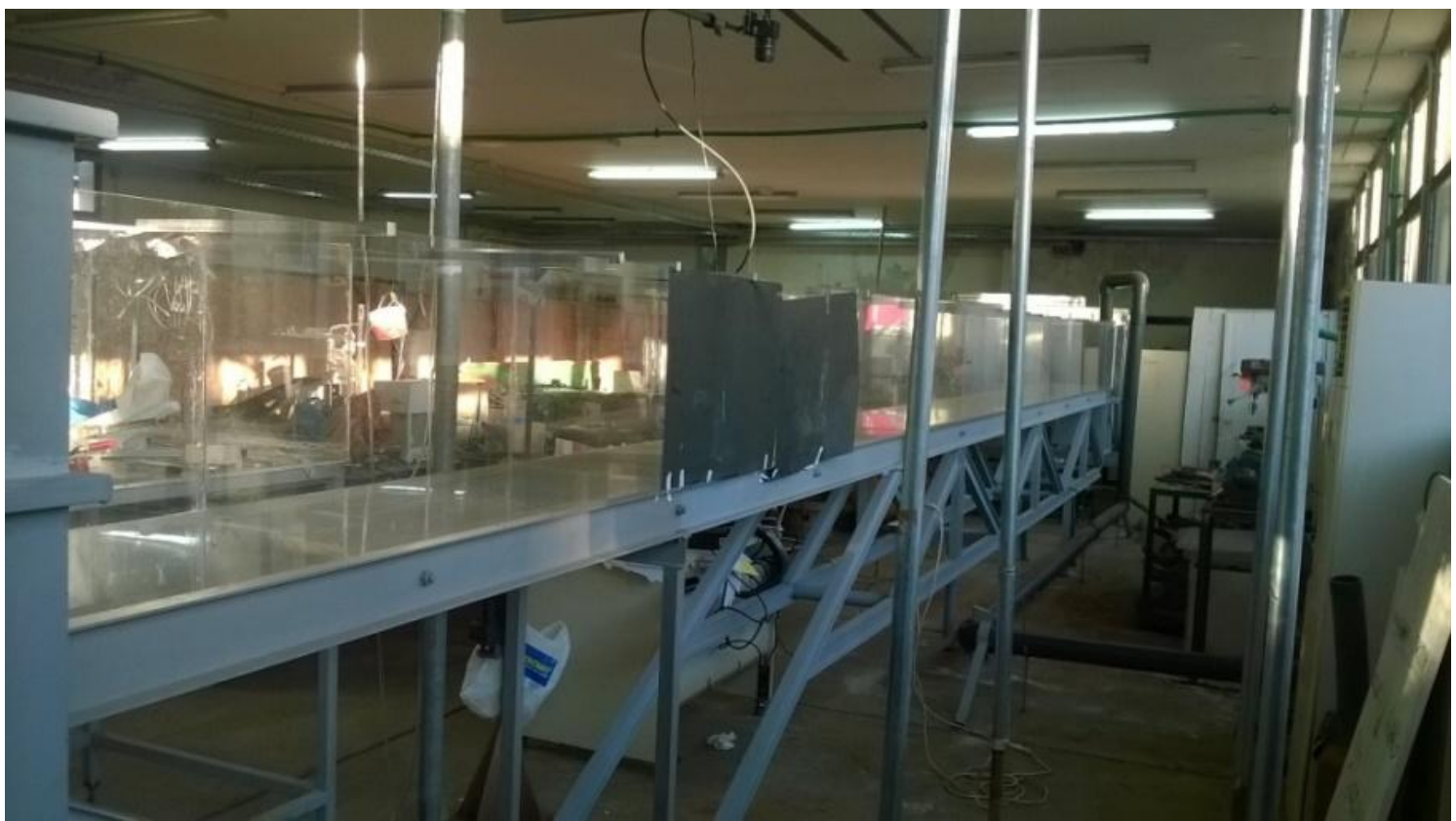

Figure 1. The NEW CHANNEL OF THE HydRAUliCS LABORATORY OF CiVIL ENG. DEPT. OF ATEITh

The mussel shocks were always taken directly from the mussel farmer and placed in the central axis of the channel leaving space up to the vertical wall of the channel around $16 \mathrm{~cm}$ from both sides (since the diameter of each shock was nearly 18 $\mathrm{cm})$. Thus, the laboratory experiments were realized using the physical scale of a shock and using current velocities in the entrance of the channel that were usually recorded in the field.

\section{MATERIAL AND METHOD}

\subsection{The experimental procedure}

The experiments were designed in physical scale and the relevant variables were determined. Moreover, the specific positions for the measurements, the depth of the flow and the velocity currents were also determined. From the aforementioned data, the appropriate mechanical and electrological mechanisms were resulted for the successful realization of the experiments. Then, the onomatology of the different experiments followed, in such way that the exact determination of each specific experiment could be resulted from the file's name. The water flowing in the channel was always collected in a tank. The level of the free surface of the water in the channel was determined by a sluice gate in the downstream part of the channel's water reservoir with accuracy of $0.5 \mathrm{~mm}$. The water would overflow from the sluice gate and finally go to a circular tank outside the room of the laboratory experiments. From there, with the help of a pump and a water pipe the water would go back to the entrance of the channel. Thus, continuous flow of recycled water was achieved with fixed hydraulic conditions. So, in this way the hydraulic parameters had the eligible fixed and invariable values while the phenomenon could be repeated at any time. The flow rate was controlled by the help of an orifice plate in the discharge pipe of the pump of the water recirculation. Downstream and upstream of the orifice plate pressure transducers were located. The difference of the indicated values in volt of the two pressure transducers was due to the flow rate and consequently from the water velocity in the channel. The pressure transducers were calibrated in order to give certain flow values corresponding to certain volt values. The following three mean velocity values were used in the experiment: $5 \mathrm{~cm} / \mathrm{sec}, 7 \mathrm{~cm} / \mathrm{sec} \mathrm{and} 9 \mathrm{~cm} / \mathrm{sec}$ corresponding to 0.5 Volt, 3 Volt and 3.5 Volt respectively. The depth of the flow was $20 \mathrm{~cm}$ and was specified with the help of a sluice gate in the downstream part of the channels' water reservoir. The sketch below depicts a ground plan of the experimental configuration. More specifically, the layout for the computation of the velocity field in front of the second mussel shock, placed at a distance L $(300,500,700$ or $900 \mathrm{~mm})$ from the first shock, is shown (Fig. 2). 


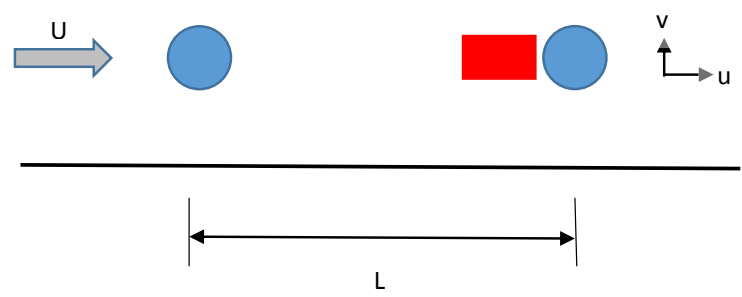

Area corresponded to the captured velocity field

Mussel Shock

\section{FIGURE 2. SKETCH SHOWING THE MUSSEL SHOCKS (BLUE CIRCLES) AND THE AREA FOR THE DESCRIPTION OF THE VELOCITY FIELD IN FRONT OF THE SECOND MUSSEL SHOCK (RED RECTANGULAR BOX) I.E. UPSTREAM OF THE SECOND SHOCK PLACED AT A DISTANCE L FROM THE FIRST MUSSEL SHOCK}

For a certain fixed entrance water velocity $\mathrm{U}$, the velocity field in the orthogonal areas of the above figure (marked in red) was determined. The blue circles in the figure correspond to the mussel shocks depicted as 1a and 2a. The shock 2a can be found either on the axis of channel's symmetry or to the lateral vertical wall of the channel. A basic research parameter used in the experiment was the distance between the shocks 1a and 2a. Four cases were taken into account: $300 \mathrm{~mm}, 500 \mathrm{~mm}$, $700 \mathrm{~mm}$ and $900 \mathrm{~mm}$. The final goal was the determination of the velocity field in the areas around the shocks.

\subsection{Measurement Technique}

The velocity field was studied with the modern technique of PIV (Particle Image Velocimetry) as described in [12]. This technique was recently used successfully by [13] who studied experimentally the effects of surrounding outer porous cylinder on vortex structure downstream of a circular inner cylinder in deep water flow. Furthermore, PIV experiments were conducted by [14] and also included in the recent research by [15]. The specific experimental procedure, adjusted in our laboratory, is described as follows: The system has two lasers which give light pulses in a controlled time (synchronizer) while the time step between two successive pulses for the velocities that we study was determined to $1.5 \mathrm{msec}$. Two photographs (twin photographs) were taken with camera CCD for each couple of pulses from the two lasers. In 1sec about 3 couples of photographs would be taken. During the realization of the experiment 200 couples of photographs were taken. From each couple a velocity field resulted. The technique of velocity calculation was based on the program INSIGHT 3G of the company TSI. The area of the photograph was divided into small orthogonal/rectangular parts -control areas (interrogation spot) with dimensions $32 \mathrm{pxX} 32 \mathrm{px}$. The distance of the camera from the section of measurements was selected so that in each $\mathrm{mm}$ should be $6.5 \mathrm{px}$ and the photographs should have dimensions $20 \mathrm{cmx} 18 \mathrm{~cm}$. From the photographs resulting from the light of the two lasers which ranged in time $1.5 \mathrm{~m} \mathrm{sec}$ and lightened the exit particles, the particle motion and transport was calculated with the help of FFT method (Fast Fourier Transform) and the Convolution Theorem. In this way the velocities $\mathrm{u}$ and $\mathrm{v}$ were computed and then the resultant velocity at each control area. The total number of the spots that the velocity value was computed was about 7300.From the measurements the instant values of flow velocity at different places were computed in time and space as well as the mean temporal and spatial velocity value. Moreover, the curves of the velocity profiles along $\mathrm{x}$ and $\mathrm{y}$ axis resulted as well as the resultant velocities. In this way the water flow around and through the shocks was described.

\section{RESUlTS}

\subsection{Distance of $\mathbf{3 0 0} \mathbf{~ m m}$}

In the case of distance between the shocks $300 \mathrm{~mm}$ the phenomenon of influence of the first mussel shock to the second shock was very intense, i.e. the generation of the Von Karman vortexes behind the first shock was influenced by the second shock: thus, conclusions for the flow could not be clear (since the flow field behind and the first shock was formed in combination with the flow field of the one in front of the second shock).

\subsection{Distance of $\mathbf{5 0 0 ~} \mathbf{~ m m}$}

The velocity field in front of the second shock, corresponding to mean flow velocity of the water at the entrance of the channel $5 \mathrm{~cm} / \mathrm{sec}$, is depicted in the following Fig. $3 \mathrm{a}$. The velocity field in front of the second shock, corresponding to mean flow velocity of the water at the entrance of the channel $7 \mathrm{~cm} / \mathrm{sec}$, is depicted in Fig. $3 \mathrm{~b}$. The velocity field in front of the 
second shock, corresponding to mean flow velocity of the water at the entrance of the channel $9 \mathrm{~cm} / \mathrm{sec}$, is depicted in the following Fig.3c.

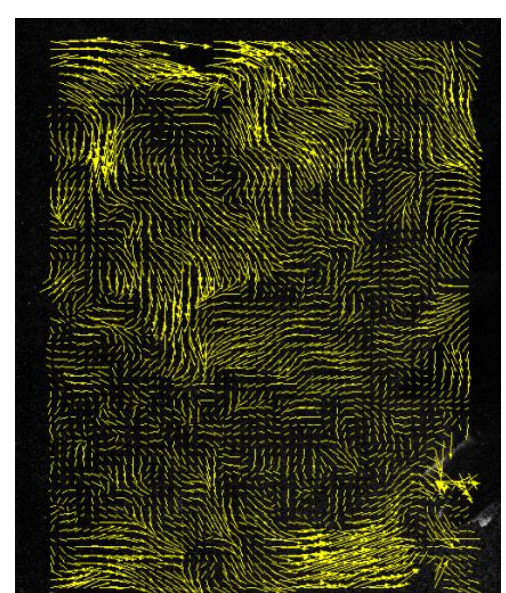

(a)

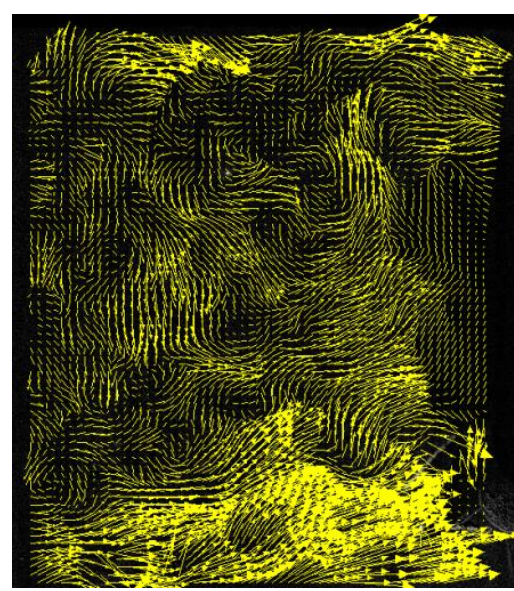

(b)

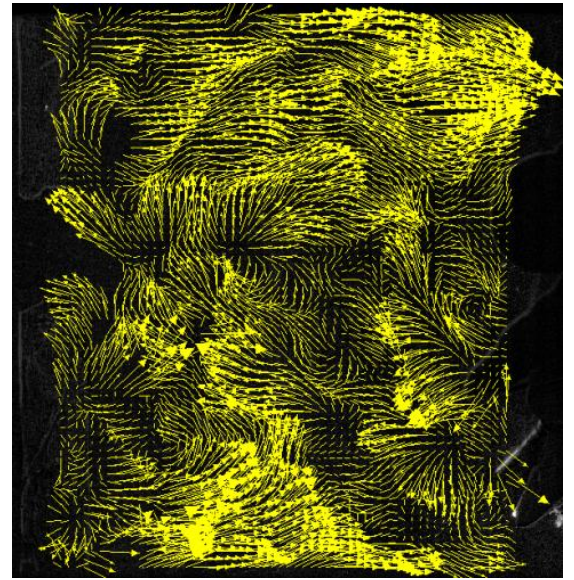

(c)

FIGURE 3: QUALITATIVE REPRESENTATION OF THE VELOCITY FIELD IN FRONT OF THE SHOCK, CORRESPONDING TO MEAN FLOW VELOCITY AT THE CHANNEL'S ENTRANCE $5 \mathrm{~cm} / \mathrm{s}(3 \mathrm{a}), 7 \mathrm{~cm} / \mathrm{s}(3 \mathrm{~b})$ and 9 $\mathrm{cm} / \mathrm{s}(3 \mathrm{c})$. The SeCONd SHOCK IS PlaCed AT A Distance OF $500 \mathrm{~mm}$ FROM THE FirSt SHOCK

Intense formation of eddies in front of each mussel shock. From the velocity fields it is clear that the vorticities seem to be intense due to spottiness of the external surface of the mussel shocks. It is this fact that causes reverse water flow.

The velocity profiles near the obstacle (mussel shock), corresponding to the three different cases of the entrance flow velocity $(5,7$ and $9 \mathrm{~cm} / \mathrm{sec})$ are given all together in Fig 4.

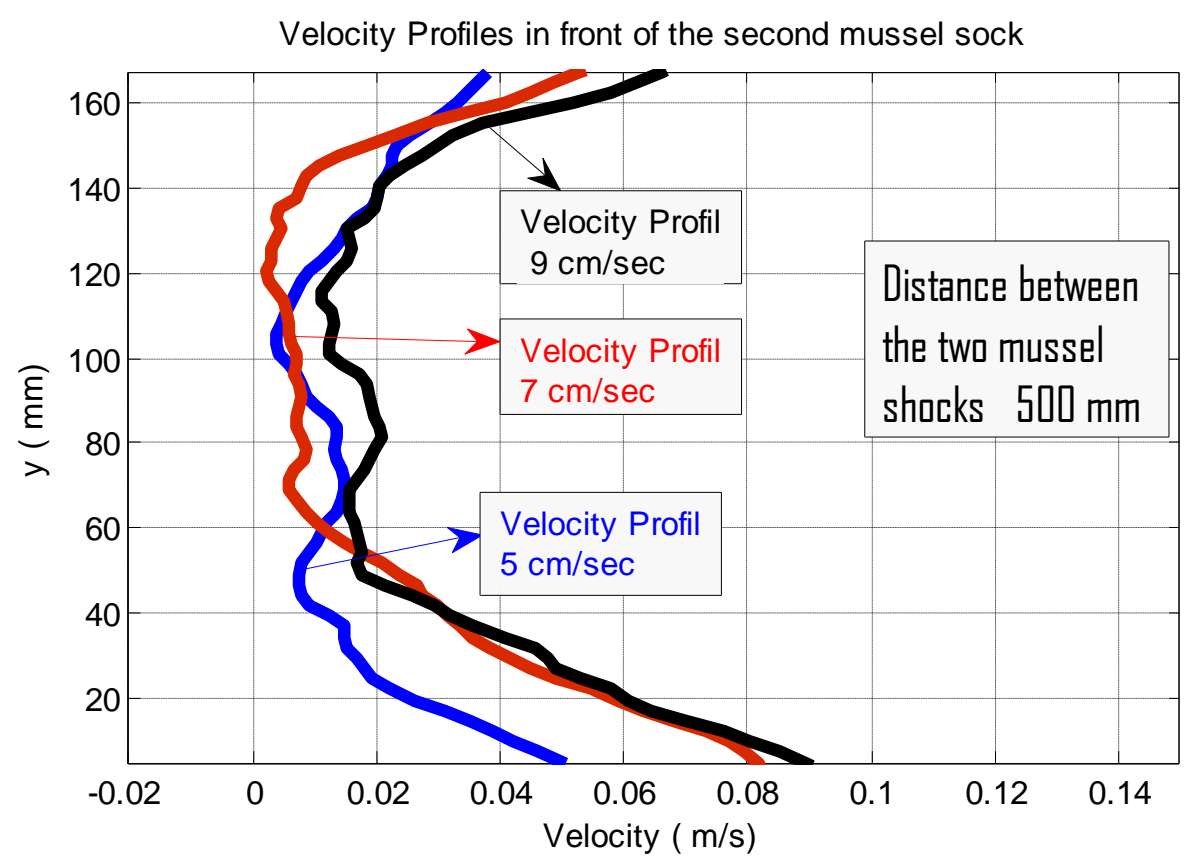

FIGURE 4: VELOCITY PROFILES IN FRONT OF THE SECOND MUSSEL SHOCK, CORRESPONDING TO ENTRANCE FLOW VELOCITIES 5, 7 and $9 \mathrm{~cm} / \mathrm{sec}$. THE SECOND SHOCK WAS PLACED AT A DISTANCE OF 500 mm FROM THE FIRST SHOCK.

The three above profiles show that as the entrance flow velocity increases the mean value of the velocity in the axis of symmetry as well as the reflected mass of water decrease. The velocity values greater than zero $(u>0)$ show that the water flow close to the shock is nearly getting zero while the larger amount of the water mass moves laterally of the shocks, i.e. in the vicinity of the channel's walls. The velocity values less than zero $(u<0)$ are due to the fact that the mussels of the shock 
create cavities and therefore return of the water mass is observed. It is noted that figure 6showsthemeaninspace (in a distance of $20 \mathrm{~mm}$ ) as well as mean in time (in a time window of $15 \mathrm{sec}$ ) velocity values.

\subsection{Distance of $700 \mathrm{~mm}$}

The velocity fields in front of the second shock, corresponding to mean flow velocities of the water at the entrance of the channel 5,7 and $9 \mathrm{~cm} / \mathrm{sec}$, is depicted in figure $5 \mathrm{a}, 5 \mathrm{~b}$ and 5 crespectively.

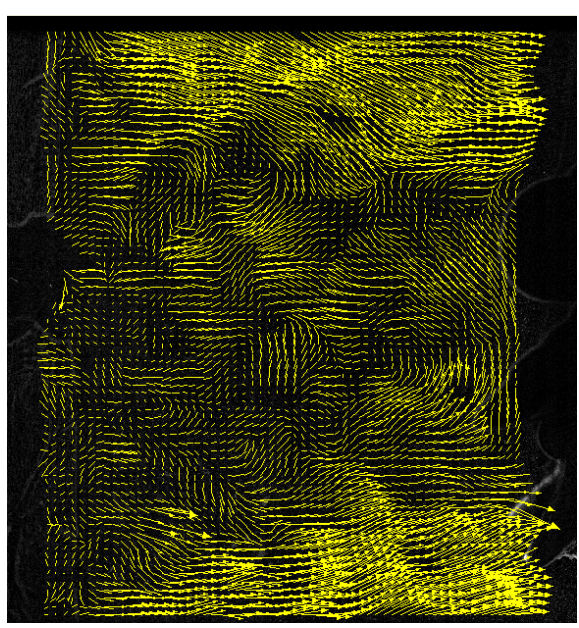

(a)

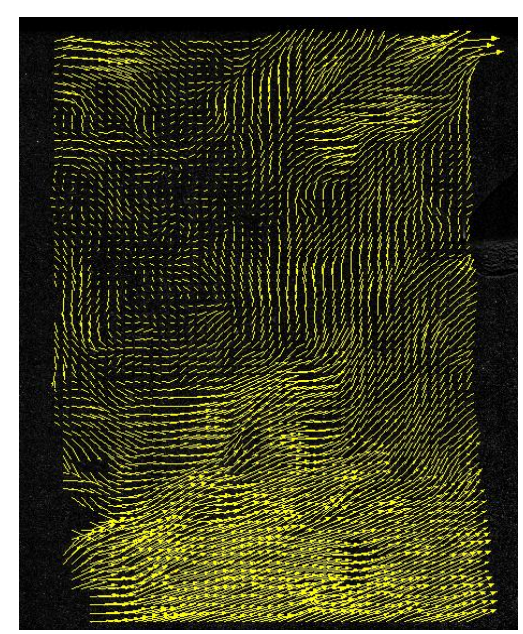

(b)

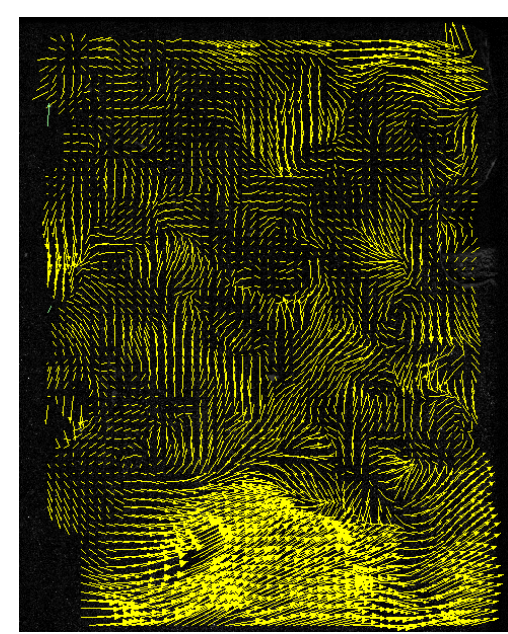

(c)

FIGURE 5: QUALITATIVE REPRESENTATION OF THE VELOCITY FIELD IN FRONT OF THE SHOCK, CORRESPONDING TO MEAN FLOW VELOCITY AT THE CHANNEL'S ENTRANCE 5 cm/s (5a), 7 cm/s (5b) and 9 $\mathrm{cm} / \mathrm{s}$ (5c). The Second Shock is Placed at A Distance of 700 mm From The First ShOCK

The velocity profiles near the mussel shock (which obviously behaves as an obstacle), corresponding to the three different cases of the entrance flow velocity $(5,7$ and $9 \mathrm{~cm} / \mathrm{sec})$ are given all together in the same plot of Fig.6.

Velocity Profiles in front of the second mussel sock

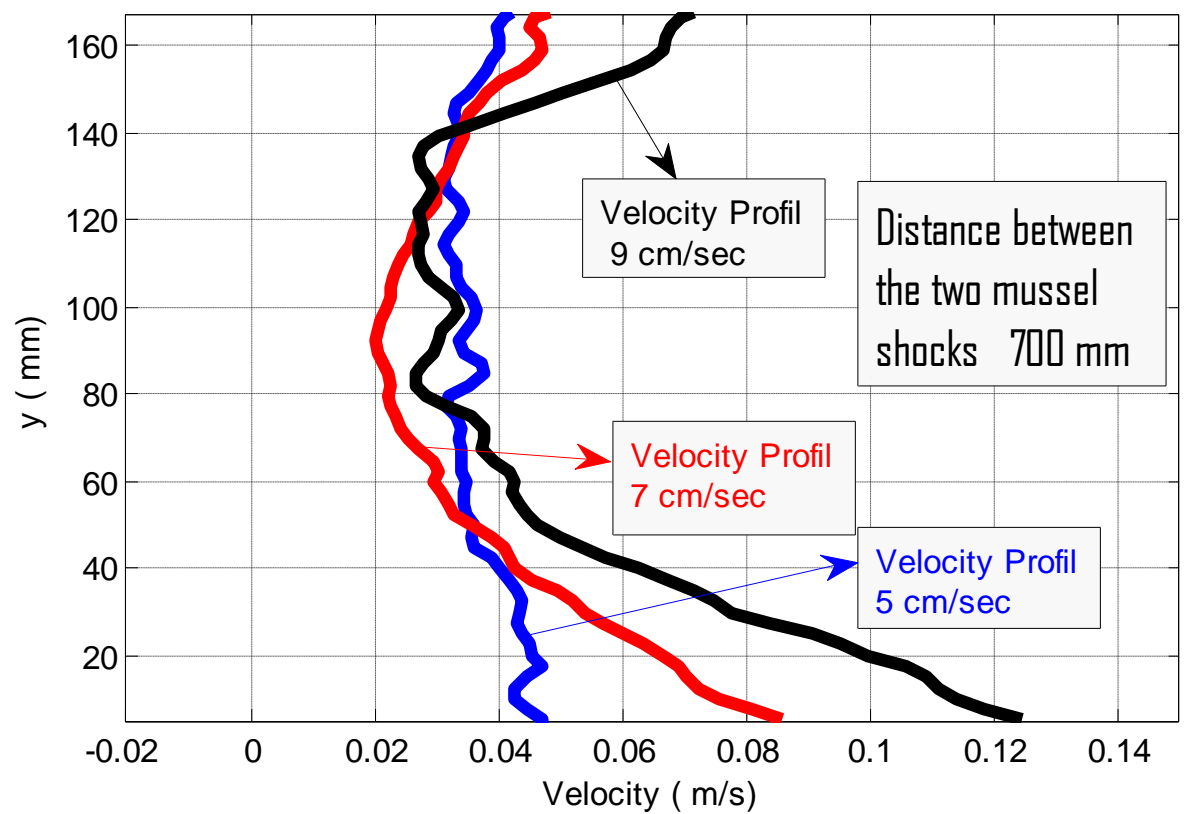

FIGURE 6: VELOCITY PROFILES IN FRONT OF THE SECOND MUSSEL SHOCK, CORRESPONDING TO ENTRANCE FLOW VELOCITIES 5, 7 and $9 \mathrm{~cm} / \mathrm{sec}$. THE SECOND SHOCK IS PLACED AT A DISTANCE OF 700 mm FROM THE FIRST SHOCK

\subsection{Distance of $900 \mathrm{~mm}$}

The velocity fields in front of the second shock, corresponding to mean flow velocities of the water at the entrance of the channel 5, 7 and $9 \mathrm{~cm} / \mathrm{sec}$, is depicted in Fig. 7a, 7b and 7c respectively. 


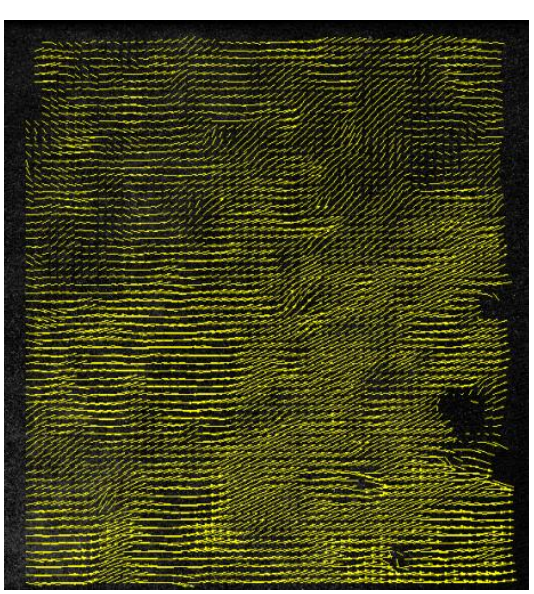

(a)

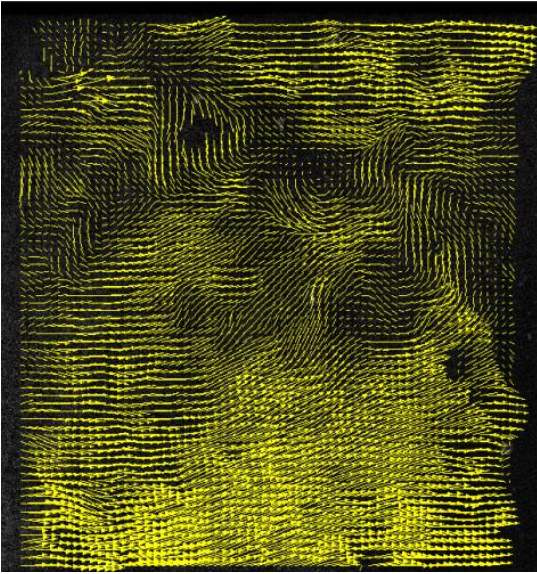

(b)

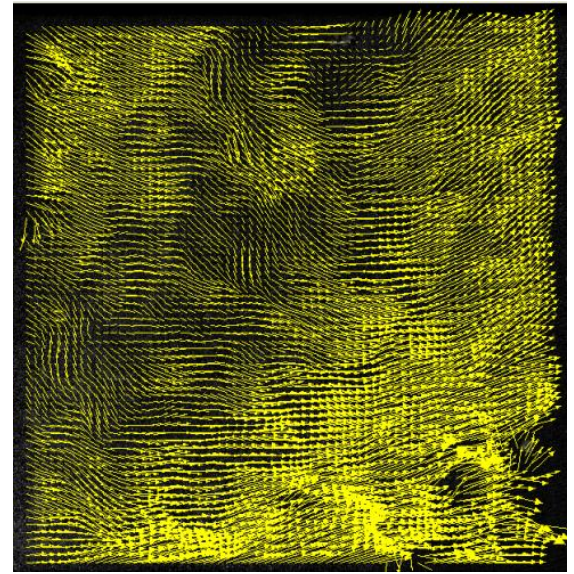

(c)

FIGURE 7: QUALITATIVE REPRESENTATION OF THE VELOCITY FIELD IN FRONT OF THE SHOCK, CORRESPONDING TO MEAN FLOW VELOCITY AT THE CHANNEL'S ENTRANCE 5 cm/s (7a), 7 cm/s (7b) and 9 $\mathrm{cm} / \mathrm{s}$ (7c). The Second Shock is Placed at A Distance OF 900 mm from The FirSt ShOCK

The velocity profiles near the mussel shock, corresponding to the three different cases of the entrance flow velocity (5, 7 and $9 \mathrm{~cm} / \mathrm{sec}$ ) are given all together in Fig 8.

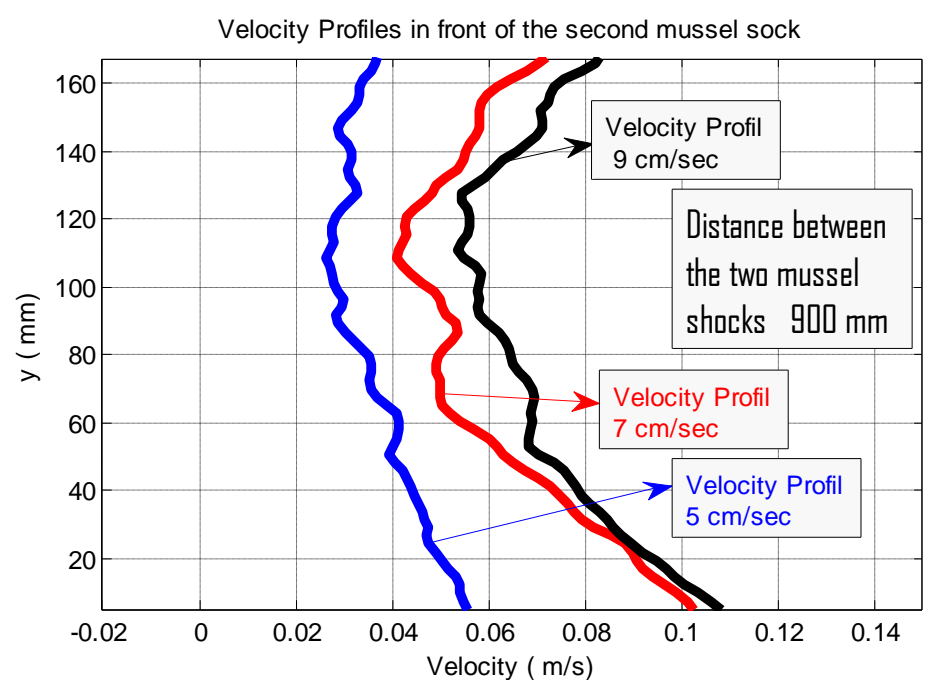

FIGURE 8: VELOCITY PROFILES IN FRONT OF THE SECOND MUSSEL SHOCK, CORRESPONDING TO ENTRANCE FLOW VELOCITIES 5, 7 AND $9 \mathrm{~cm} / \mathrm{sec}$. THE SECOND SHOCK IS PLACED AT A DISTANCE OF 900 mm FROM THE FIRST SHOCK.

It is interesting here to note that figure 8 shows that the case of larger distance between the shocks (i.e. $90 \mathrm{~cm}$ )presents the largest percentage of the velocity class $5-10 \mathrm{~cm} / \mathrm{sec}$ that seems to occur for initial entrance current velocities 7 and $9 \mathrm{~cm} / \mathrm{s}$, which is in agreement with field observations reported by Savvidis et al. 2015 and already commented in the introduction. Also, it is observed that for distance between the shocks $900 \mathrm{~mm}$, the differences of velocity profiles is quite more distinct for the different entrance current velocities 5,7 and $9 \mathrm{~cm} / \mathrm{sec}$.

The velocity profiles $\mathrm{U}$ along the flow direction (axis $\mathrm{x}$ ) (with the normal axis corresponding to $\mathrm{V}$ velocities) show that the velocities are characterized by intense changes due to the irregularities of the mussels' surface. More specifically, values $>0$ and $<0$ observed in the field lead to the conclusion that negligible or minimal flow is developing through the body of the mussels. Consequently the mussel shock behaves as a nearly solid (rigid) body.

\subsection{Further issues to be checked}

The low velocities close to the shocks raised the question if there is flow through the body of the mussel shocks and for that reason another one experiment was realized for the control of the flow though the shock. The photo below (Fig 9) shows the confirmation that there is minimal flow inside and through the body of the mussel shocks. 


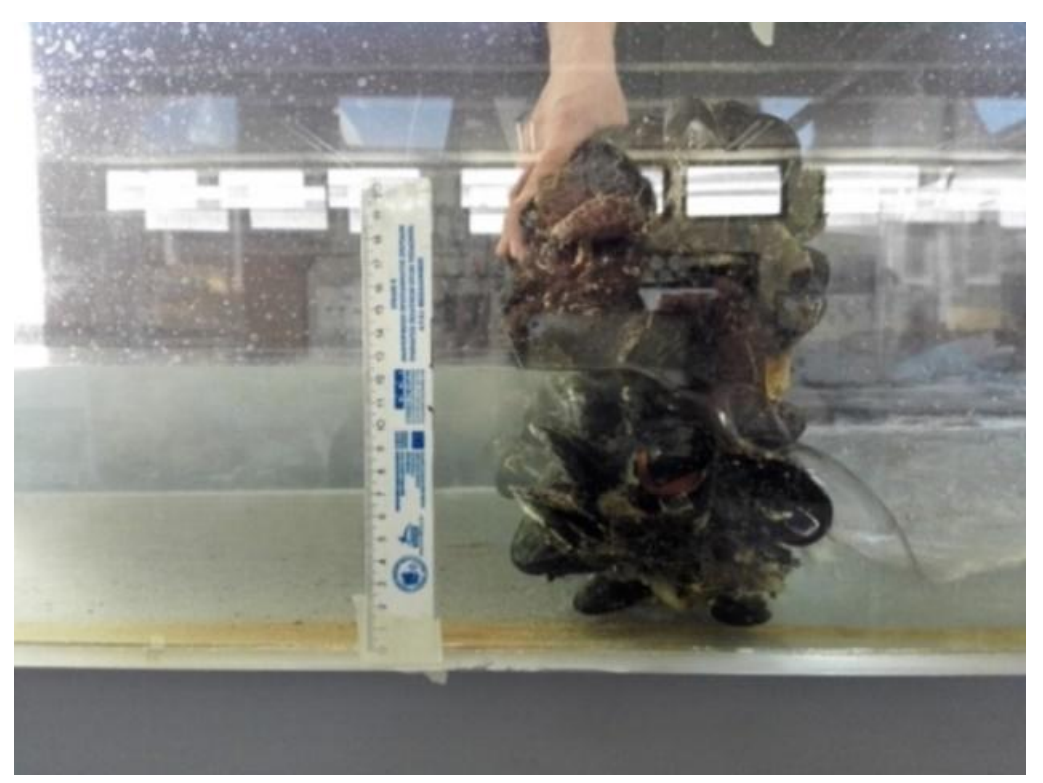

\section{FIGURE 9: ADDITIONAL EXPERIMENTS FOR THE FLOW UPSTREAM AND DOWNSTREAM OF THE MUSSEL SHOCK}

Two shocks were located normally to the flow direction so that the water flow could be investigated. For mean velocity $10 \mathrm{~cm} / \mathrm{sec}$ rise of the surface water level upstream about $12.5 \mathrm{~cm}$ while the surface water level downstream was $2.5 \mathrm{~cm} / \mathrm{sec}$. The observed flow was due to the spaces in-between the two shocks. The head loss (drop of the pressure) of the $10 \mathrm{~cm}$ from the upstream position to the downstream position does not seem to allow flow through the body of the mussel shocks, or at least allows negligible flow. Moreover, if we take into account the fact that the hydraulic head of $5 \mathrm{~cm}$ corresponds to velocity $1 \mathrm{~m} / \mathrm{sec}$, while the water velocity in our experiment is $0.1 \mathrm{~m} / \mathrm{sec}$, then we can conclude that there minimal flow through the body of the mussel shocks, while the main flow seems to occur through the spaces between the two shocks (the upper and the lower ones). This conclusion is enhanced from the observation of the flow during the realization of the experiment.

\section{CONCLUSIONS}

The following conclusions have been raised from the experimental procedure and the analysis of the data collected.

a) For the distance between the mussel shocks 500 and $700 \mathrm{~mm}$ the velocity profiles (fig.6ka110) seem to have the same values in the central area of the shock for the different entrance (initial) current velocities with small divergences concerning the velocity profiles for initial current velocity $9 \mathrm{~cm} / \mathrm{sec}$

b) For distance between the shocks $900 \mathrm{~mm}$, the distinction (difference) of velocity profiles is more clear (intense) for the different entrance (initial) current velocities. Moreover the case of larger distance between the shocks (i.e. $90 \mathrm{~cm}$ ) seems to present the largest percentage of the velocity class $5-10 \mathrm{~cm} / \mathrm{sec}$ that seems to occur for initial entrance current velocities 7 and $9 \mathrm{~cm} / \mathrm{s}$, which is in line with previous research findings in the field.

c) For shock distances between the shocks greater than $500 \mathrm{~mm}$ the velocity field is almost restored. This is obvious from the pattern of the velocity fields and the velocity profiles.

d) The comparison of the diagrams with the velocity profiles show that the increase of distance between the shocks leads to increase of the velocity values. For values of the initial current velocity $5 \mathrm{~cm} / \mathrm{sec}$ the velocity profiles for distances 700 and $900 \mathrm{~mm}$ nearly coincide

e) From the velocity fields it is clear that the vorticities seem to be intense due to spottiness of the external surface of the mussel shocks. It is this fact that causes reverse water flow.

f) In large values of the mean water velocities, large returning velocities are observed while the resultant velocities (mean values in time and space) are decreasing significantly close to the mussel shocks. Those mean values close to the shocks are of the order of $1 \mathrm{~cm} / \mathrm{sec}$ or below this value.

g) Intense formation of eddies in front of each mussel shock is observed. 


\section{ACKNOWLEDGMENTS}

Acknowledgement: SUCCESS project has received funding from the European Union's H2020 program under grant agreement No 635188.

\section{REFERENCES}

[1] National Center of Marine Research (NCMR) (2001), "Management Study of the Zones of Mussel Production in Thessaloniki and Thermaikos Bays". Final Report for Fisheries Directorate of Thessaloniki Prefecture, Supervisor E. Papathanasiou, pp. 147

[2] Sofia Galinou -Mitsoudi, Yiannis Savvidis and Xenofon Dimitriadis, (2006). 'Interaction between mussel cultures and hydrodynamics; a preliminary study in Thessaloniki \& Thermaikos Gulfs' Journal of Biological Research, Vol. 6, pp. 139-145

[3] Savvidis, Y., Antoniou, A., Dimitriadis, X., Moriki, A., Galinou - Mitsoudi, S. Alvanou L., Petridis, D. and Koutitas, C., (2007). "Hydrodynamics in a mussel culture area in Thermaikos gulf" in Proceedings of the 8th International Conference on the Mediterranean Coastal Environment, MEDCOAST 07. Alexandria, Egypt, 1263-1274.

[4] Moriki A, Galinou-Mitsoudi S, Petridis D, Kosti D, Savvidis Y, Dimitriadis X, Koutitas C, Alvanou L (2008), "Environmental impacts of intensive mussel culture in the coastal waters of the gulf of Thessaloniki (N. Greece)". Fresenius Environmental Bulletin, 17, 1945-1955

[5] Savvidis Yiannis, Alexandros Antoniou, Vasilis-Orestis Stoilas and Sofia Galinou-Mitsoudi (2015) "Hydrodynamics in longline mussel culture layouts" 12h International Conference on the Mediterranean Coastal Environment MEDCOAST 2015, Varna, Bulgaria, 6-10 October 2015

[6] Konstantinou Z, Savvidis Y., Kombiadou K. and Krestenitis Y. (2011), "Mussel farm layout scenarios as management alternatives",In: Ozhan, E. (Ed),Proceedings of the Tenth International Conference on the Mediterranean Coastal Environment MEDCOAST 2011, 25-29October, Rhodes, Greece, 735-746.

[7] Konstantinou, Z. (2013), "Integrated Coastal Management through the Utilization of Conceptual and Numerical Tools" Ph.D Thesis, Department of Civil Engineering, Aristotle University of Thessaloniki, Greece (in Greek).

[8] Inglis GJ, Hayden BJ, Ross AH (2000), “An overview of factors affecting the carrying capacity of coastal embayments for mussel culture",NIWA client report, National Institute for Water and Atmospheric Research Ltd, New Zealand, p. 38.

[9] David Russell Plew (2005) The Hydrodynamic Effects of Long-line Mussel Farms. PhD thesis Department of Civil Engineering, University of Canterbury, Christchurch, New Zealand

[10] DEHKORDI Behzad Ghadiri, MOGHADDAM Hesam Sarvghad, JAFARI Hamed Houri (2011) NUMERICAL SIMULATION OF FLOW OVER TWO CIRCULAR CYLINDERS IN TANDEM ARRANGEMENT. Journal of Hydrodynamics 2011,23(1):114-126, DOI: 10.1016/S1001-6058(10)60095-9

[11] A. F. Abdel Gawad, N. A. Mohamed, S. A. Mohamed, M. S. Matbuly (2013) Investigation of the channel flow with internal obstacles using large eddy simulation and finite-element technique, Applied and Computational Mathematics, 2013; 2(1) : 1-13

[12] Adrian Ronald J., (1991) PARTICLE-IMAGING TECHNIQUES FOR EXPERIMENTAL FLUID MECHANICS, Annu. Rev. Fluid Mech. 1991, $23: 261304$

[13] B. Gozmen, E. Firat, H. Akilli and B. Sahin (2017) Flow control behind a circular cylinder via a porous cylinder in deep water EPJ Web of Conferences

DOI: 10.1051/C_Owned by the authors, published by EDP Sciences, 2013, epjconf201 / 01035 (2013) 4534501035

[14] Keramaris E, Pechlivanidis G (2013) PIV measurements over a permeable and an impermeable bed, J. of Porous Media 16(1):21-28.

[15] Savvidis Y., E. Keramaris, Pechlivanidis G and C. Koutitas (2017) Optimum Design of the Entrance of a Fishpond Laterally to the Main Stream of an Open Channel, Environmental Science and Pollution Research, DOI: 10.1007/s11356-017-8696-z 